\title{
Quark Mass Relations to Four-Loop Order in Perturbative QCD
}

\author{
Peter Marquard, ${ }^{1}$ Alexander V. Smirnov, ${ }^{2}$ Vladimir A. Smirnov, ${ }^{3}$ and Matthias Steinhauser ${ }^{4}$ \\ ${ }^{1}$ Deutsches Elektronen Synchrotron DESY, Platanenallee 6, 15738 Zeuthen, Germany \\ ${ }^{2}$ Scientific Research Computing Center, Moscow State University, 119991 Moscow, Russia \\ ${ }^{3}$ Skobeltsyn Institute of Nuclear Physics, Moscow State University, 119991 Moscow, Russia \\ ${ }^{4}$ Institut für Theoretische Teilchenphysik, Karlsruhe Institute of Technology (KIT), 76128 Karlsruhe, Germany
}

(Received 7 February 2015; published 7 April 2015)

\begin{abstract}
We present results for the relation between a heavy quark mass defined in the on-shell and minimal subtraction $(\overline{\mathrm{MS}})$ scheme to four-loop order. The method to compute the four-loop on-shell integral is briefly described and the new results are used to establish relations between various short-distance masses and the $\overline{\mathrm{MS}}$ quark mass to next-to-next-to-next-to-leading order accuracy. These relations play an important role in the accurate determination of the $\overline{\mathrm{MS}}$ heavy quark masses.
\end{abstract}

PACS numbers: 12.38.Bx, 12.38.Cy, 14.65.Fy, 14.65.Ha

The precise knowledge of quark masses plays an important role in many phenomenological applications. This is in particular true for the heavy top, bottom, and charm quarks. For example, the top quark mass enters as a crucial parameter the combined electroweak fits which have been used to obtain indirect information about the Higgs boson mass, and nowadays serve as consistency checks for the standard model, see, e.g., Refs. [1,2]. The uncertainty in the top quark mass is also dominant in the analyses of the stability of the electroweak vacuum [3-5]. A prominent example where a precise bottom quark mass is required are $B$-meson decays which are often proportional to the fifth power of $m_{b}$. Precise charm and bottom quark masses are important to obtain accurate predictions for the Higgs boson decays into the respective quark flavors. Also in the context of top and bottom Yukawa coupling unification, precise mass values are indispensable since they serve as boundary conditions at low energies. Last but not least, quark masses enter the Lagrange density of the standard model as fundamental parameters. Thus, it is mandatory to obtain precise numerical values by comparing high-order theoretical predictions with precise experimental data.

At lowest order in perturbation theory there is no need to fix the renormalization scheme for the quark masses. However, after including quantum corrections it is necessary to apply renormalization conditions which fix the renormalization scheme. A natural scheme for heavy quark masses, i.e., the charm, bottom, and top quark masses, is the on-shell (OS) scheme where one requires that the inverse heavy quark propagator with momentum $q$ has a zero at the position of the on-shell mass, $M$, i.e., for $q^{2}=M^{2}$. It is well known that perturbation theory has a bad convergence behavior in case the on-shell quark mass is used as a parameter. Another widely used renormalization scheme is based on minimal subtraction. This means that the mass parameter entering the quark propagator is defined in such a way that just divergent terms (and no finite contributions) are absorbed such that the quark propagator is finite (after wave function renormalization). In this Letter we consider four-loop corrections to the relation between the on-shell and the minimal subtraction $(\overline{\mathrm{MS}})$ definition of a heavy quark mass that allows for a precise conversion from one renormalization scheme into the other.

For the various heavy quarks, different methods relying on different quark mass definitions are used to extract the mass values. For example, in Ref. [6] low-moment sum rules have been used to extract directly the $\overline{\mathrm{MS}}$ charm and bottom quark masses without any reference to the on-shell mass. On the other hand, physical observables inherently connected to the threshold, like $\Upsilon$ sum rules or top quark pair production close to threshold, rely on properly defined quark masses, like the potential subtracted (PS) [7], 1S [8-10], or renormalon subtracted (RS) [11] definition. When comparing with experimental data, in a first step the corresponding mass values are extracted. Afterwards, they are converted to the $\overline{\mathrm{MS}}$ definition. Note that the relation between the $\overline{\mathrm{MS}}$ and the OS mass is an important ingredient to obtain the relation between the PS, 1S, or RS masses and the $\overline{\mathrm{MS}}$ mass.

In this Letter we use the four-loop $\overline{\mathrm{MS}}$-OS relation to establish relations between the PS, 1S, RS and the $\overline{\mathrm{MS}}$ quark mass, which are necessary to obtain the latter with next-to-next-to-next-to-leading order $\left(\mathrm{N}^{3} \mathrm{LO}\right)$ accuracy.

Note that there is a further definition of a threshold mass, the so-called kinetic mass [12] which has been used for quite a number of applications in $B$ physics, (see, e.g., Ref. [13]). However, the relation to the on-shell mass is only known to two loops (NNLO). For this reason it is not considered in the following.

In the following we first discuss the relation between the $\overline{\mathrm{MS}}$ and OS quark mass. Afterwards we elaborate on the relation between the threshold (PS, 1S, and RS) and the $\overline{\mathrm{MS}}$ mass. The latter is obtained by using as starting point the definition of the PS, 1S, or RS masses which establishes a 
relation to the pole mass. Afterwards the pole mass is replaced by the $\overline{\mathrm{MS}}$ mass which leads to the desired relation between the short-distance masses.

To derive a formula relating the $\overline{\mathrm{MS}}$ and OS quark mass it is advantageous to start with relations between theses masses and the bare mass, $m^{0}$, which are given by (we refrain from adding a superscript "OS" to the on-shell mass but use a capital letter. Similarly, a lowercase $m$ without further superscript stands for the $\overline{\mathrm{MS}}$ quark mass. For all other mass definitions we use a lowercase " $m$ " and a superscript indicating the renormalization scheme.)

$$
m^{0}=Z_{m}^{\overline{\mathrm{MS}}} m, \quad m^{0}=Z_{m}^{\mathrm{OS}} M
$$

$Z_{m}^{\overline{\mathrm{MS}}}$ is known to four loops and can be found in Refs. [14-16]. By construction, the ratio of the two equations in (1) is finite which leads to

$$
z_{m}(\mu)=\frac{m(\mu)}{M},
$$

where $z_{m}$ depends on $\alpha_{s}(\mu)$ and $\log (\mu / M)$ and has the following perturbative expansion

$$
z_{m}(\mu)=\sum_{n \geq 0}\left(\frac{\alpha_{s}}{\pi}\right)^{n} z_{m}^{(n)},
$$

with $z_{m}^{(0)}=1$.

For a derivation of convenient formulas relating $z_{m}(\mu)$ to the on-shell quark self energy we refer to Refs. [17-19] where it is shown that $Z_{m}^{\mathrm{OS}}$ is obtained from the sum of scalar and vector contribution evaluated on-shell, i.e.,

$$
Z_{m}^{\mathrm{OS}}=1+\Sigma_{V}\left(q^{2}=M^{2}\right)+\Sigma_{S}\left(q^{2}=M^{2}\right) .
$$

One-, two-, and three-loop QCD results to $Z_{m}^{\mathrm{OS}}$ have been computed in Refs. [17,20] and [18,19,21,22], respectively, and electroweak effects have been considered in Refs. [23-27]. The main task of this Letter is the computation of the four-loop QCD corrections to $Z_{m}^{\mathrm{OS}}$ and, consequently, to $z_{m}$. For convenience we introduce also the inverse relation to Eq. (2) as follows:

$$
M=m(\mu) c_{m}(\mu) .
$$

The PS quark mass has been introduced in Ref. [7]. Its relation to the pole mass is given by

$$
m^{\mathrm{PS}}=M-\delta m\left(\mu_{f}\right),
$$

with

$$
\delta m\left(\mu_{f}\right)=-\frac{1}{2} \int_{|\vec{q}|<\mu_{f}} \frac{d^{3} q}{(2 \pi)^{3}} V(\vec{q}),
$$

where $V(\vec{q})$ is the perturbative contribution to the static heavy quark potential. $\delta m\left(\mu_{f}\right)$ can be computed in perturbative QCD and has the form

$$
\begin{aligned}
\delta m\left(\mu_{f}\right)= & \mu_{f} \frac{C_{F} \alpha_{s}}{\pi}\left\{1+\frac{\alpha_{s}}{4 \pi}\left[a_{1}+\beta_{0}\left(2+\log \frac{\mu^{2}}{\mu_{f}^{2}}\right)\right]\right. \\
& +\cdots\},
\end{aligned}
$$

where $\beta_{0}=11-2 n_{l} / 3$ is the one-loop coefficient of the QCD $\beta$ function and $a_{1}=31 / 3-10 n_{l} / 9$ the one-loop coefficient of the static potential. $n_{l}$ is the number of massless quarks. $\mu_{f}$ is the factorization scale which is of the order of the soft scale. In this Letter we use $\mu_{f}=2 \mathrm{GeV}$ for bottom and $\mu_{f}=20 \mathrm{GeV}$ for top quarks. $\delta m\left(\mu_{f}\right)$ is known to $\mathrm{N}^{3} \mathrm{LO}$ [28], which involves the three-loop corrections to the static potential, $a_{3}$ [29-31].

The $\mathrm{N}^{3} \mathrm{LO}$ relation between $m^{\mathrm{PS}}$ and $m$ is obtained by inserting Eq. (5) into Eq. (6). All ingredients are already expanded and the coefficients of $\left(\alpha_{s}\right)^{n}$ have simply to be combined. In particular, the term in Eq. (6) involving $a_{3}$ is combined with the four-loop term in the $m-M$ relation. One obtains an explicit formula to compute the PS mass in case the $\overline{\mathrm{MS}}$ is given. For a given PS mass we solve this equation iteratively to obtain the $\overline{\mathrm{MS}}$ mass.

The $1 \mathrm{~S}$ mass is defined as half the perturbative mass of a fictitious $1^{3} S_{1}$ state, where it is assumed that the quark is stable. Thus, we have the following relation between the $1 \mathrm{~S}$ and on-shell mass [8-10]

$$
m^{1 \mathrm{~S}}=M+\left.\frac{1}{2} E_{1}^{\mathrm{pt}}\right|_{\alpha_{s}^{n} \rightarrow \alpha_{s}^{n} \varepsilon^{n-1}},
$$

where $E_{1}^{\mathrm{pt}}$ is the perturbative ground state energy which is available to third order $[28,32,33]$. The last missing ingredient was the three-loop static potential which has been evaluated in Refs. [29-31]. The replacement $\alpha_{s}^{n} \rightarrow$ $\alpha_{s}^{n} \varepsilon^{n-1}$ implements the so-called $\varepsilon$ expansion which guarantees that the appropriate orders in the expansions of $E_{1}^{\mathrm{pt}}$ and $M$ (in terms of $m$ ) are combined.

The perturbative expansion of $E_{1}^{\mathrm{pt}}$ has the following form

$$
\left.E_{1}^{\mathrm{pt}}\right|_{\alpha_{s}^{n} \rightarrow \alpha_{s}^{n} \varepsilon^{n-1}}=-\varepsilon \frac{C_{F}^{2} M \alpha_{s}^{2}}{8} \sum_{n \geq 0}\left(\varepsilon \frac{\alpha_{s}}{\pi}\right)^{n} \delta_{E}^{(n)} .
$$

In order to obtain the relation between $m^{1 \mathrm{~S}}$ and $m$ one has to replace $\alpha_{s}^{\left(n_{l}+1\right)}$ in Eq. (5) by $\alpha_{s}^{\left(n_{l}\right)}$ and then apply the replacement $\alpha_{s}^{n} \rightarrow \alpha_{s}^{n} \varepsilon^{n}$. Afterwards it is inserted into Eq. (9) and expanded in the parameter $\varepsilon$. This guarantees that the $\mathrm{N}^{k} \mathrm{LO}$ term in $E_{1}^{\mathrm{pt}}$ is combined with the $(k+1)$ loop correction to $c_{m}(\mu)$. In particular, in order to establish the $m^{1 \mathrm{~S}}-m$ relation to $\mathrm{N}^{3} \mathrm{LO}$ four-loop corrections to $c_{m}(\mu)$ are needed. A given $\overline{\mathrm{MS}}$ quark mass is transformed to the $1 \mathrm{~S}$ mass by inserting the numerical value into the resulting 
equation. In case the $1 \mathrm{~S}$ mass is given one obtains the $\overline{\mathrm{MS}}$ mass by solving the equation implicitly.

A further threshold mass, the so-called RS mass, has been introduced in Ref. [11]. It is related to the pole mass in such a way that the pure renormalon contributions are subtracted. The corresponding formulas are derived and explicitly given in Ref. [11]. In that reference also, a variant, the so-called $\mathrm{RS}^{\prime}$ scheme, is discussed where no subtraction is performed for the $\mathcal{O}\left(\alpha_{s}\right)$ term in the $\overline{\mathrm{MS}}-\mathrm{OS}$ relation. Recently the numerical accuracy of the normalization constant of the first renormalon has been improved in Ref. [34], where also a variant of the $\mathrm{RS}$ and $\mathrm{RS}^{\prime}$ masses has been suggested in which (in the case of the bottom quark) the subtraction term is parameterized in terms of $\alpha_{s}^{(3)}$. In this Letter we will adopt the prescription of Ref. [11]. Similarly to the PS mass also for the RS mass a subtraction scale has to be specified which we again choose as $\mu_{f}=2 \mathrm{GeV}$ for bottom and $\mu_{f}=20 \mathrm{GeV}$ for top quarks.

For the computation of the scalar and vector part of the fermion propagator we use an automated setup which generates all contributing amplitudes, processes them with FORM3 [35] and provides scalar functions involving several million different integrals encoded in functions with 14 different indices that belong to 100 different integral families.

The Laporta algorithm [36] is applied to each family using FIRE5 [37] and CRUSHER [38], which are written in $\mathrm{C}++$. Then we use the code TSORT [39], which is part of the latest FIRE version, to reveal relations between primary master integrals following recipes of [40] and end up with 386 four-loop massive on-shell propagator integrals, i.e., with $p^{2}=M^{2}$.

We have performed the calculation allowing for a general gauge parameter $\xi$ keeping terms up to order $\xi^{2}$ in the expression we give to the reduction routines. We have checked that $\xi$ drops out after mass renormalization but before inserting the master integrals.

For some master integrals, analytic results could be derived using a straightforward loop-by-loop integration for general space-time dimension. We also used analytical results obtained for nontrivial four-loop on-shell master integrals computed in our earlier paper, Ref. [41]. In some other cases one- and twofold Mellin-Barnes representations can be derived which allow for a high-precision numeric evaluation, at least up to 20 digits. For some of the master integrals, we applied threefold MB representations which enabled us to obtain a precision of eight digits.

For factorizable integrals, we obtained analytic results from known two- and three-loop results. In particular, we used Ref. [42] where the expansion in $\epsilon=(4-d) / 2$ has been performed up to the order typical to four-loop calculations. ( $d$ is the space-time dimension used to compute the momentum integrals.)
We computed the remaining 332 integrals numerically with the help of FIESTA [43-45]. FIESTA returns for each $\epsilon$ coefficient a numerical result and the corresponding uncertainty from the numerical integration. When inserting the master integrals we keep track of all uncertainties and combine them quadratically in the final expression. We interpret the resulting uncertainty as a standard deviation and multiply it by five in the final result for the relation between the $\overline{\mathrm{MS}}$ and OS quark mass. This is in agreement with adding the uncertainties from the individual contributions linearly.

We are now in the position to present numerical results for $z_{m}(\mu)$ which have been obtained by setting the number of colors to three $\left(N_{c}=3\right)$ and the number of massless quarks $\left(n_{l}\right)$ to either 3,4 , or 5 , corresponding to the charm, bottom, or top quark case, before combining the uncertainties from the numerical integration of the master integrals. Note that the coefficients up to three loops are known analytically $[18,19]$. We refrain from listing the corresponding results but refer to Eq. (13) of Ref. [46]. Analytical results are also available for the logarithmic four-loop contributions since they can easily be obtained using renormalization group methods. In the following we restrict ourselves to compact numerical results. At four loops we obtain for the coefficient of $\left(\alpha_{s} / \pi\right)^{4}$

$$
\begin{aligned}
\left.z_{m}^{(4)}\right|_{n_{l}=3}= & -1744.8 \pm 21.5-703.48 l_{\mathrm{OS}}-122.97 l_{\mathrm{OS}}^{2} \\
& -14.234 l_{\mathrm{OS}}^{3}-0.75043 l_{\mathrm{OS}}^{4}, \\
\left.z_{m}^{(4)}\right|_{n_{l}=4}= & -1267.0 \pm 21.5-500.23 l_{\mathrm{OS}}-83.390 l_{\mathrm{OS}}^{2} \\
& -9.9563 l_{\mathrm{OS}}^{3}-0.514033 l_{\mathrm{OS}}^{4}, \\
\left.z_{m}^{(4)}\right|_{n_{l}=5}= & -859.96 \pm 21.5-328.94 l_{\mathrm{OS}}-50.856 l_{\mathrm{OS}}^{2} \\
& -6.4922 l_{\mathrm{OS}}^{3}-0.33203 l_{\mathrm{OS}}^{4},
\end{aligned}
$$

with $l_{\mathrm{OS}}=\ln \left(\mu^{2} / M^{2}\right)$. We obtain the $\mu$-independent coefficients with an accuracy of $1.2 \%$ for $n_{l}=3,1.7 \%$ for $n_{l}=4$ ) and $2.5 \%$ for $n_{l}=5$. In the numerical results discussed below we will assume a relative uncertainty of $3 \%$ for all values of $n_{l}$.

For convenience we also show the four-loop results for $c_{m}$ which read

$$
\begin{aligned}
\left.c_{m}^{(4)}\right|_{n_{l}=3}= & 1691.2 \pm 21.5+828.43 l_{\overline{\mathrm{MS}}}+189.65 l_{\overline{\mathrm{MS}}}^{2} \\
& +36.688 l_{\overline{\mathrm{MS}}}^{3}+4.8124 l_{\overline{\mathrm{MS}}}^{4}, \\
\left.c_{m}^{(4)}\right|_{n_{l}=4}= & 1224.0 \pm 21.5+601.98 l_{\overline{\mathrm{MS}}}+134.10 l_{\overline{\mathrm{MS}}}^{2} \\
& +28.846 l_{\overline{\mathrm{MS}}}^{3}+3.9648 l_{\overline{\mathrm{MS}}}^{4}, \\
\left.c_{m}^{(4)}\right|_{n_{l}=5}= & 827.37 \pm 21.5+408.88 l_{\overline{\mathrm{MS}}}+86.574 l_{\overline{\mathrm{MS}}}^{2} \\
& +22.023 l_{\overline{\mathrm{MS}}}^{3}+3.2227 l_{\overline{\mathrm{MS}}}^{4},
\end{aligned}
$$


with $l \overline{\mathrm{MS}}=\ln \left(\mu^{2} / \mathrm{m}^{2}\right)$. In the remaining part of this Letter we will concentrate on the top and bottom quark mass.

As an application of the new results in Eqs. (11) and (12) we study the relations between the various threshold masses and the $\overline{\mathrm{MS}}$ mass. We use the following input values for the strong coupling constant and the bottom and top quark masses $[6,47,48]$ :

$$
\begin{aligned}
\alpha_{s}^{(5)}\left(M_{Z}\right) & =0.1185, \quad m_{b}\left(m_{b}\right)=4.163 \mathrm{GeV}, \\
M_{t} & =173.34 \mathrm{GeV} .
\end{aligned}
$$

$\alpha_{s}$ with four and six active flavors is obtained from $\alpha_{s}^{(5)}$ where for the decoupling scale we choose twice the heavy quark mass $[46,49]$.

Let us have a closer look to the relation between the OS and $\overline{\mathrm{MS}}$ top quark mass. For $\mu=m_{t}$ we have

$$
\begin{aligned}
M_{t}= & m_{t}\left(1+0.4244 \alpha_{s}+0.8345 \alpha_{s}^{2}+2.375 \alpha_{s}^{3}\right. \\
& \left.+(8.49 \pm 0.25) \alpha_{s}^{4}\right) \\
= & 163.643+7.557+1.617+0.501 \\
& +0.195 \pm 0.005 \mathrm{GeV}
\end{aligned}
$$

with $\alpha_{s} \equiv \alpha_{s}^{(6)}\left(m_{t}\right)=0.1088$. Note that the four-loop term still gives a contribution of about $200 \mathrm{MeV}$ which is not negligible even with nowadays uncertainties from TEVATRON and LHC [48]. The corresponding results for the bottom quark read

$$
\begin{aligned}
M_{b}= & m_{b}\left(1+0.4244 \alpha_{s}+0.9401 \alpha_{s}^{2}+3.045 \alpha_{s}^{3}\right. \\
& \left.+(12.57 \pm 0.38) \alpha_{s}^{4}\right) \\
= & 4.163+0.401+0.201+0.148 \\
& +0.138 \pm 0.004 \mathrm{GeV} .
\end{aligned}
$$

Here, $\alpha_{s} \equiv \alpha_{s}^{(5)}\left(m_{b}\right)=0.2268$. Note that the four-loop corrections in Eq. (15) are almost as large as the threeloop term. On the other hand, the perturbative series for the case of the top quark has a reasonable behavior: the threeloop coefficient is by a factor three smaller than the twoloop one and the four-loop term is again smaller by a factor 2.5. This suggests that with the help of Eq. (14) the top quark mass can be determined with an uncertainty below $200 \mathrm{MeV}$.

In practice it often happens that in a first step a threshold quark mass is extracted from comparisons of higher order calculations and experimental measurements. Afterwards the threshold mass is converted to the MS quark mass. In Tables I and II we show the results for the scale invariant $\overline{\mathrm{MS}}$ quark mass $m_{q}\left(m_{q}\right)(q=b, t)$ using one- to four-loop accuracy for the conversion.

In the case of the top quark (cf. Table I) the three-loop corrections amount to about $200-250 \mathrm{MeV}$ which reduces
TABLE I. $m_{t}\left(m_{t}\right)$ in GeV computed from the PS, 1S, and RS quark mass using one- to four-loop accuracy. The numbers in the last line are obtained by increasing the four-loop coefficient in Eq. (12) by $3 \%$.

\begin{tabular}{lccc}
\hline \hline No. of loops & $m^{\mathrm{PS}}=171.792$ & $m^{1 \mathrm{~S}}=172.227$ & $m^{\mathrm{RS}}=171.215$ \\
\hline 1 & 165.097 & 165.045 & 164.847 \\
2 & 163.943 & 163.861 & 163.853 \\
3 & 163.687 & 163.651 & 163.663 \\
4 & 163.643 & 163.643 & 163.643 \\
$4(\times 1.03)$ & 163.637 & 163.637 & 163.637 \\
\hline \hline
\end{tabular}

to $\{44,8,20\} \mathrm{MeV}$ at four loops for the $\{\mathrm{PS}, 1 \mathrm{~S}, \mathrm{RS}\}$ quark mass. A $3 \%$ uncertainty in the $\overline{\mathrm{MS}}-\mathrm{OS}$ relation induces a shift of $6 \mathrm{MeV}$ in $m_{t}\left(m_{t}\right)$ which is in general small as compared to the four-loop contribution. Let us estimate the final uncertainty from the conversion to the $\overline{\mathrm{MS}}$ mass from the quadratic combination of the $6 \mathrm{MeV}$ with half of the four-loop contribution (i.e., $\{44,8,20\} \times \frac{1}{2} \mathrm{MeV}$ ). This leads to $\{23,7,11\} \mathrm{MeV}$ which should be added in quadrature to the remaining uncertainties of the threshold mass.

The results for $m_{b}\left(m_{b}\right)$ computed from the PS, $1 \mathrm{~S}$, and RS threshold masses are shown in Table II. The three-loop corrections provide still sizable effects of up to $40 \mathrm{MeV}$ which reduces to at most $9 \mathrm{MeV}$ at four loops. The uncertainty in the four-loop $\overline{\mathrm{MS}}$-OS relation induces an error of $4 \mathrm{MeV}$. Thus we arrive at a final error of $\{4,6,5\} \mathrm{MeV}$ for the conversion from the $\{1 \mathrm{~S}, \mathrm{PS}, \mathrm{RS}\}$ mass. This is not negligible, though in general much smaller than other uncertainties involved in the quark mass extraction (see, e.g., Refs. [34,50] and [51] for recent determinations of $m_{b}\left(m_{b}\right)$ where in intermediate steps the $1 \mathrm{~S}, \mathrm{RS}$, and PS has been used, respectively).

The results of Tables I and II can be used, in combination with similar calculations for different values of $\alpha_{s}\left(M_{Z}\right)$ and threshold masses, to construct the following approximation formulas

$$
\begin{aligned}
& \frac{m_{t}\left(m_{t}\right)}{\mathrm{GeV}}=163.643 \pm 0.023+0.074 \Delta_{\alpha_{s}}-0.095 \Delta_{m_{t}}^{\mathrm{PS}}, \\
& \frac{m_{t}\left(m_{t}\right)}{\mathrm{GeV}}=163.643 \pm 0.007+0.069 \Delta_{\alpha_{s}}-0.096 \Delta_{m_{t}}^{1 \mathrm{~S}}, \\
& \frac{m_{t}\left(m_{t}\right)}{\mathrm{GeV}}=163.643 \pm 0.011+0.067 \Delta_{\alpha_{s}}-0.095 \Delta_{m_{t}}^{\mathrm{RS}}, \\
& \frac{m_{b}\left(m_{b}\right)}{\mathrm{GeV}}=4.163 \pm 0.004+0.007 \Delta_{\alpha_{s}}-0.018 \Delta_{m_{b}}^{\mathrm{PS}}, \\
& \frac{m_{b}\left(m_{b}\right)}{\mathrm{GeV}}=4.163 \pm 0.006+0.008 \Delta_{\alpha_{s}}-0.019 \Delta_{m_{b}}^{1 \mathrm{~S}}, \\
& \frac{m_{b}\left(m_{b}\right)}{\mathrm{GeV}}=4.163 \pm 0.005+0.004 \Delta_{\alpha_{s}}-0.018 \Delta_{m_{b}}^{\mathrm{RS}}
\end{aligned}
$$


TABLE II. $m_{b}\left(m_{b}\right)$ in GeV computed from the PS, $1 \mathrm{~S}$, and RS quark mass using one- to four-loop accuracy. The numbers in the last line are obtained by increasing the four-loop coefficient in Eq. (12) by $3 \%$.

\begin{tabular}{lccc}
\hline \hline No. of loops & $m^{\mathrm{PS}}=4.483$ & $m^{1 \mathrm{~S}}=4.670$ & $m^{\mathrm{RS}}=4.365$ \\
\hline 1 & 4.266 & 4.308 & 4.210 \\
2 & 4.191 & 4.190 & 4.172 \\
3 & 4.161 & 4.154 & 4.158 \\
4 & 4.163 & 4.163 & 4.163 \\
$4(\times 1.03)$ & 4.159 & 4.159 & 4.159 \\
\hline \hline
\end{tabular}

TABLE III. Comparison of predictions for the four-loop coefficient $c_{m}^{(4)}$ to our result which is present in the last line.

\begin{tabular}{llll}
\hline \hline & \multicolumn{3}{c}{$c_{m}^{(4)}[\mu=m(m)]$} \\
\cline { 2 - 4 } Ref. & $n_{l}=3$ & $n_{l}=4$ & $n_{l}=5$ \\
\hline$[52]$ & 1668 & 1324 & 1031 \\
{$[53]$} & 1571.4 & 1107.8 & 727.0 \\
{$[54]$} & 1281 & 986 & 719 \\
{$[55]$} & 1785.9 & 1316.4 & 920.1 \\
{$[56]$} & $1668 \pm 167$ & $1258_{-66}^{+26}$ & $897_{-175}^{+31}$ \\
{$[34]$} & $1772 \pm 82$ & $1324 \pm 82$ & $945 \pm 92$ \\
this work & $1691.2 \pm 21.5$ & $1224.0 \pm 21.5$ & $827.37 \pm 21.5$ \\
\hline \hline
\end{tabular}

with $\quad \Delta_{\alpha_{s}}=\left[0.1185-\alpha_{s}\left(M_{Z}\right)\right] / 0.001, \quad \Delta_{m_{t}}^{\mathrm{PS}}=$ $\left(171.792 \mathrm{GeV}-m_{t}^{\mathrm{PS}}\right) / 0.1, \quad \Delta_{m_{t}}^{1 \mathrm{~S}}=(172.227 \mathrm{GeV}-$ $\left.m_{t}^{1 \mathrm{~S}}\right) / 0.1, \quad \Delta_{m_{t}}^{\mathrm{RS}}=\left(171.215 \mathrm{GeV}-m_{t}^{\mathrm{RS}}\right) / 0.1, \quad \Delta_{m_{b}}^{\mathrm{PS}}=$ $\left(4.483 \mathrm{GeV}-m_{b}^{\mathrm{PS}}\right) / 0.02, \quad \Delta_{m_{b}}^{1 \mathrm{~S}}=\left(4.670 \mathrm{GeV}-m_{b}^{1 \mathrm{~S}}\right) /$ $0.02, \Delta_{m_{b}}^{\mathrm{RS}}=\left(4.365 \mathrm{GeV}-m_{b}^{\mathrm{RS}}\right) / 0.02$.

Let us finally compare in Table III our result for the fourloop coefficient $c_{m}^{(4)}$ to predictions obtained on the basis of different assumptions. In general good agreement is found, in particular, with the results from Refs. $[34,55,56]$ which are all based on renormalon cancellation. For example, in Ref. [56], the four-loop coefficient is extracted from the requirement of perturbative stability of the combination $2 m_{\text {pole }}+V_{\mathrm{QCD}}$, where $V_{\mathrm{QCD}}$ is the static potential of two heavy quarks. The estimates in Ref. [53] have been obtained only on the basis of the two-loop results, leading, nevertheless, to good approximations. Somewhat lower results have been obtained in Ref. [54] where dispersive methods have been used and large $\pi^{2}$ terms have been identified. In Ref. [52] the four-loop relation between the on-shell and $\overline{\mathrm{MS}}$ quark mass has been estimated using the large $\beta_{0}$ approximation.

To conclude, in this Letter we have computed the fourloop corrections between the on-shell and $\overline{\mathrm{MS}}$ definition of heavy quarks. Our main results are given in Eqs. (11) and (12) for charm, bottom, and top quarks. As applications we have derived precise relations between the PS, 1S, and RS threshold masses and the $\overline{\mathrm{MS}}$ quark mass.

We are thankful to Tobias Huber for advice on numerical evaluation of Mellin-Barnes integrals. M. S. would like to thank Konstantin Chetyrkin, Hans Kühn, and Jan Piclum for useful discussions. We thank Konstantin Chetyrkin and Kirill Melnikov for useful comments to the manuscript. We thank the High Perfomance Computing Center Stuttgart (HLRS) and the Supercomputing Center of Lomonosov Moscow State University [57] for providing computing time used for the numerical computations with FIESTA. P. M. was supported in part by the EU Network HIGGSTOOLS PITN-GA-2012-316704. This work was supported by the DFG through the SFB/TR 9 "Computational Particle Physics." The work of V.S. was supported by the Alexander von Humboldt Foundation (Humboldt Forschungspreis).

[1] S. Schael et al. (ALEPH and DELPHI and L3 and OPAL and LEP Electroweak Collaborations), Phys. Rep. 532, 119 (2013).

[2] M. Ciuchini, E. Franco, S. Mishima, and L. Silvestrini, J. High Energy Phys. 08 (2013) 106.

[3] F. Bezrukov, M. Y. Kalmykov, B. A. Kniehl, and M. Shaposhnikov, J. High Energy Phys. 10 (2012) 140.

[4] G. Degrassi, S. Di Vita, J. Elias-Miro, J. R. Espinosa, G. F. Giudice, G. Isidori, and A. Strumia, J. High Energy Phys. 08 (2012) 098.

[5] S. Alekhin, A. Djouadi, and S. Moch, Phys. Lett. B 716, 214 (2012).

[6] K. G. Chetyrkin, J. H. Kühn, A. Maier, P. Maierhofer, P. Marquard, M. Steinhauser, and C. Sturm, Phys. Rev. D 80, 074010 (2009).

[7] M. Beneke, Phys. Lett. B 434, 115 (1998).

[8] A. H. Hoang, Z. Ligeti, and A. V. Manohar, Phys. Rev. D 59, 074017 (1999).

[9] A. H. Hoang, Z. Ligeti, and A. V. Manohar, Phys. Rev. Lett. 82, 277 (1999).

[10] A. H. Hoang and T. Teubner, Phys. Rev. D 60, 114027 (1999).

[11] A. Pineda, J. High Energy Phys. 06 (2001) 022.

[12] A. Czarnecki, K. Melnikov, and N. Uraltsev, Phys. Rev. Lett. 80, 3189 (1998).

[13] P. Gambino and C. Schwanda, Phys. Rev. D 89, 014022 (2014).

[14] K. G. Chetyrkin, Phys. Lett. B 404, 161 (1997).

[15] J. A. M. Vermaseren, S. A. Larin, and T. van Ritbergen, Phys. Lett. B 405, 327 (1997).

[16] K. G. Chetyrkin, Nucl. Phys. B710, 499 (2005).

[17] N. Gray, D. J. Broadhurst, W. Grafe, and K. Schilcher, Z. Phys. C 48, 673 (1990).

[18] K. Melnikov and T. v. Ritbergen, Phys. Lett. B 482, 99 (2000).

[19] P. Marquard, L. Mihaila, J. H. Piclum, and M. Steinhauser, Nucl. Phys. B773, 1 (2007).

[20] R. Tarrach, Nucl. Phys. B183, 384 (1981).

[21] K. G. Chetyrkin and M. Steinhauser, Phys. Rev. Lett. 83, 4001 (1999).

[22] K. G. Chetyrkin and M. Steinhauser, Nucl. Phys. B573, 617 (2000).

[23] R. Hempfling and B. A. Kniehl, Phys. Rev. D 51, 1386 (1995). 
[24] F. Jegerlehner and M. Y. Kalmykov, Nucl. Phys. B676, 365 (2004).

[25] M. Faisst, J. H. Kühn, and O. Veretin, Phys. Lett. B 589, 35 (2004).

[26] S. P. Martin, Phys. Rev. D 72, 096008 (2005).

[27] D. Eiras and M. Steinhauser, J. High Energy Phys. 02 (2006) 010.

[28] M. Beneke, Y. Kiyo, and K. Schuller, Nucl. Phys. B714, 67 (2005).

[29] A. V. Smirnov, V. A. Smirnov, and M. Steinhauser, Phys. Lett. B 668, 293 (2008).

[30] A. V. Smirnov, V. A. Smirnov, and M. Steinhauser, Phys. Rev. Lett. 104, 112002 (2010).

[31] C. Anzai, Y. Kiyo, and Y. Sumino, Phys. Rev. Lett. 104, 112003 (2010).

[32] A. A. Penin and M. Steinhauser, Phys. Lett. B 538, 335 (2002).

[33] Y. Kiyo and Y. Sumino, Nucl. Phys. B889, 156 (2014).

[34] C. Ayala, G. Cvetic, and A. Pineda, J. High Energy Phys. 09 (2014) 045.

[35] J. A. M. Vermaseren, arXiv:math-ph/0010025.

[36] S. Laporta, Int. J. Mod. Phys. A 15, 5087 (2000).

[37] A. V. Smirnov, Comput. Phys. Commun. 189, 182 (2015).

[38] P. Marquard and D. Seidel (unpublished).

[39] A. Pak, J. Phys. Conf. Ser. 368, 012049 (2012).

[40] A. V. Smirnov and V. A. Smirnov, Comput. Phys. Commun. 184, 2820 (2013).

[41] R. Lee, P. Marquard, A. V. Smirnov, V. A. Smirnov, and M. Steinhauser, J. High Energy Phys. 03 (2013) 162.

[42] R. N. Lee, A. V. Smirnov, and V. A. Smirnov, Nucl. Phys. B856, 95 (2012).
[43] A. V. Smirnov and M. N. Tentyukov, Comput. Phys. Commun. 180, 735 (2009).

[44] A. V. Smirnov, V. A. Smirnov, and M. Tentyukov, Comput. Phys. Commun. 182, 790 (2011).

[45] A. V. Smirnov, Comput. Phys. Commun. 185, 2090 (2014).

[46] K. G. Chetyrkin, J. H. Kühn, and M. Steinhauser, Comput. Phys. Commun. 133, 43 (2000).

[47] K. A. Olive et al. (Particle Data Group Collaboration), Chin. Phys. C 38, 090001 (2014).

[48] (ATLAS and CDF and CMS and D0 Collaborations), arXiv:1403.4427.

[49] B. Schmidt and M. Steinhauser, Comput. Phys. Commun. 183, 1845 (2012).

[50] A. Hoang, P. Ruiz-Femenia, and M. Stahlhofen, J. High Energy Phys. 10 (2012) 188.

[51] M. Beneke, A. Maier, J. Piclum, and T. Rauh, Nucl. Phys. B891, 42 (2015).

[52] M. Beneke and V. M. Braun, Phys. Lett. B 348, 513 (1995).

[53] K. G. Chetyrkin, B. A. Kniehl, and A. Sirlin, Phys. Lett. B 402, 359 (1997).

[54] A. L. Kataev and V. T. Kim, Phys. Part. Nucl. 41, 946 (2010).

[55] C. Ayala and G. Cvetic, Phys. Rev. D 87, 054008 (2013).

[56] Y. Sumino, Phys. Lett. B 728, 73 (2014).

[57] V. Sadovnichy, A. Tikhonravov, V. Voevodin, and V. Opanasenko, 'Lomonosov': Supercomputing at Moscow State University. In Contemporary High Performance Computing: From Petascale toward Exascale (Chapman \& Hall/CRC Computational Science, Boca Raton, USA, 2013), p. 283. 